\title{
轻核 $\alpha$ 团簇结构理论和实验研究进展
}

\author{
曹喜光 ${ }^{1} ， 马$ 余刚 ${ }^{(12) *}$
}

(1) 中国科学院上海应用物理研究所, 上海 201800;

(2) 上海科技大学物质科学与技术学院, 上海 200031

* 联系人, E-mail: ygma@sinap.ac.cn

2014-12-09 收稿, 2015-01-06 接受, 2015-05-29 网络版发表

国家自然科学基金委员会创新研究群体科学基金(11421505)、国家重点基础研究发展计划(2014CB845401, 2013CB834405)和国家自然科学 基金青年基金项目(11305239)资助

摘要 $\alpha$ 团簇在轻 $\alpha$ 共轭核基态及激发态中广泛存在, 轻核的 $\alpha$ 团簇结构不仅对核天体物 理中核合成过程和元素丰度具有决定性影响, 而且这些态的精确数据还成为检验各种新 型多体理论和核力的天然实验室。本文简要总结了描述 $\alpha$ 团簇的不同理论方法, 尤其比较 了这些不同方法对 ${ }^{12} \mathrm{C}$ Hoyle态的最新研究结果, 各种理论都支持Hoyle态是一个具有较大 尺寸的团簇结构, 但对 $\alpha$ 团簇在Hoyle态中的存在形式一一比如 $\alpha$ 凝聚, $\alpha$ 气体或是 $\alpha$ 晶体结 构, 目前还没有达成共识; 进一步从实验角度介绍了 $\alpha$ 团簇衰变的高精度符合测量及转动 态的集体激发. 对 $\alpha$ 团簇结构尤其 ${ }^{12} \mathrm{C}$ Hoyle态及 ${ }^{16} \mathrm{O}$ 类Hoyle态中 $\alpha$ 存在形式的确认需要深 入理解这些团簇态的集体激发行为, 任何实质性进展不仅高度依赖高精度实验数据, 而且 需要新的多体理论解释, 而这其中需要合理考虑核子-核子、团簇-团簇关联及连续谱的 影响.

对 $\beta$ 稳定线附近核的基态，壳结构占主导地位, 但对 $N=Z$ 偶数核(也就是 $\alpha$ 共轭核)的一些基态、激发态 及远离 $\beta$ 稳定线的核中, 团簇结构广泛存在, 存在着 壳结构和团簇结构的相互竞争. 早在1938年 Hafstad 和Teller ${ }^{[1]}$ 通过对 $\alpha$ 共轭核, 比如 ${ }^{8} \mathrm{Be},{ }^{12} \mathrm{C},{ }^{16} \mathrm{O}$ 等基态 结合能的分析得出结合能和 $\alpha$ 团簇之间键的个数成 正比, 暗示这些核基态中存在 $\alpha$ 笛结构并且 $\alpha-\alpha 之$ 间相互作用的能量是固定的. 后来对核结构的单粒 子描述变得流行起来, 现在看来这些对 $\alpha$ 共轭核的基 态描述过于简化, 但对后来核团簇理论的发展开启 了先河. 30 年后Ikeda和他的合作者 ${ }^{[2]}$ 提出 $\alpha$ 共轭核中 出现 $\alpha$ 团簇结构的阈值条件, 即著名的Ikeda图, 如图 1(a)所示. Ikeda图指出在团簇衰变阈值附近 $\alpha$ 共轭核 会出现各种团簇分量. 比如 ${ }^{8} \mathrm{Be}$ 基态可以看做 2 个 $\alpha$ 构 成的非束缚态, 在 $2 \alpha$ 衰变阈值之上 $92 \mathrm{keV}$, 具体出现
那种团簇依赖于系统的激发能 ${ }^{[3]}$. 除了轻核, 相比于 Tseng等人 ${ }^{[4]}$ 的质量公式, Ren等人 ${ }^{[5]}$ 利用结合能对 $\alpha$ 关联的研究暗示在中重核中也可能存在 $\alpha$ 团簇自由 度，另外他们对中子滴线附近 $N=2 Z$ 核基态结合能的 分析发现其中可能存在氚团簇结构 ${ }^{[6]}$. 最近, Ebran等 人 $^{[7]}$ 和Freer ${ }^{[8]}$ 还讨论了势阱深度对 $\alpha$ 团簇形成及存在 形式的影响. $\alpha$ 团簇在 $N=\mathrm{Z}$ 的偶数核中起主导作用, 一方面因为 $\alpha$ 比其周边的核具有更大的结合能, 另一 方面依赖于 $\alpha$ 非常高的第一激发态 $(20.2 \mathrm{MeV})$, 这2 个条件使 $\alpha$ 可以成为一个惰性的子单元，这些 $\alpha$ 粒子 的特殊性质可追溯到强相互作用的具体细节. 对于 $N \neq \mathrm{Z}$ 核, 推广的Ikeda图 ${ }^{[9]}$ 预言多余的中子会起到像 分子中的共价键类似的功能，使不同的团簇结合在 一起, 如图1(b)所示, 这些中子被称作共价中子. 它 们对分子态中的关联起着主导作用, 影响着这些核的 

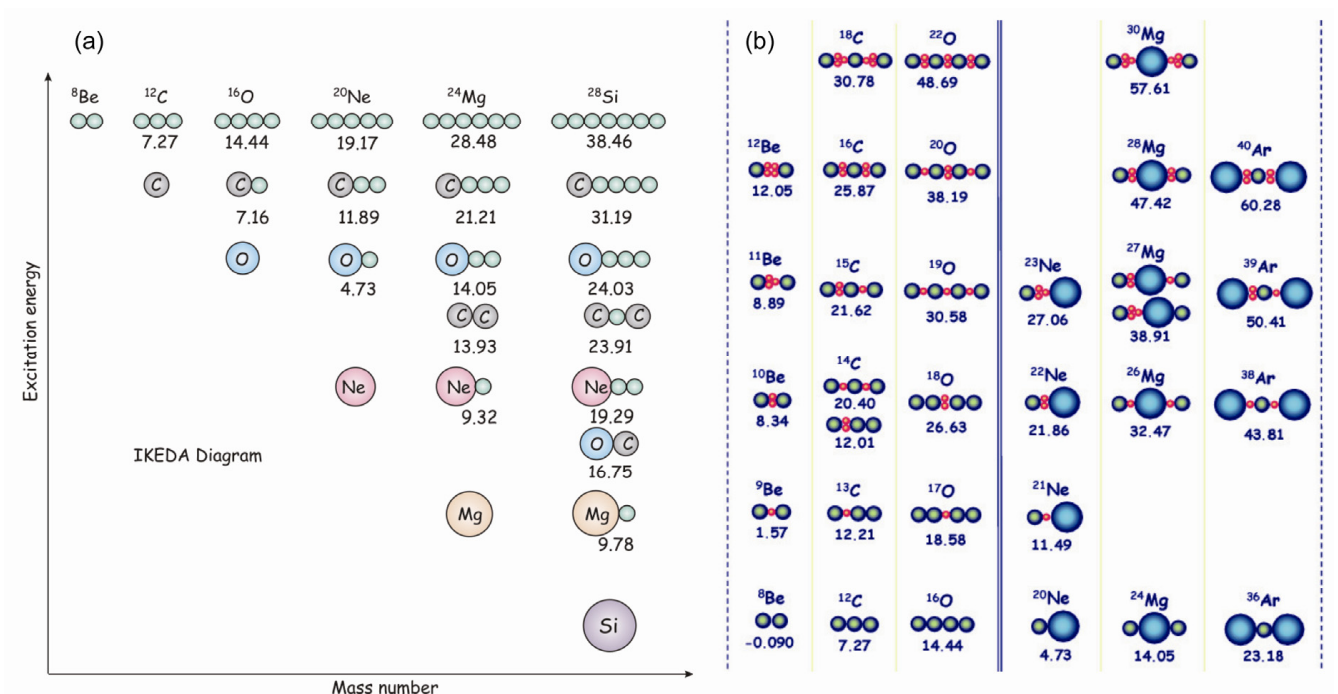

图 1 (网络版彩色)理论预言的从 ${ }^{8} \mathrm{Be}$ 到 ${ }^{40} \mathrm{Ar}$ 核的 Ikeda 阈值图. (a) 标准的 Ikeda 阈值图 ${ }^{[2]}$, 预言在 $\alpha$ 共轭核中, 在团簇衰变阈值附近, 会出现 团簇自由度; (b) 扩展的 Ikeda 阈值图 ${ }^{[9]}$, 预言在非 $\alpha$ 共轭核中, 在团簇衰变阈值附近, 共价中子对核中团簇自由度的影响

Figure 1 (Color online) Ikeda threshold diagram for the nuclei from ${ }^{8} \mathrm{Be}$ to ${ }^{40} \mathrm{Ar}$. (a) Standard Ikeda threshold diagram ${ }^{[2]}$, predicting that degree of freedom of $\alpha$ will emerge near cluster decay threshold in $\alpha$-conjugate nuclei; (b) modified Ikeda threshold diagram ${ }^{[9]}$, predicting that covalent neutron will play a role near cluster decay threshold in non- $\alpha$-conjugate nuclei

形状及形变. 如何从理论上描述、理解这些结构, 以 及实验上的证实将对核物理的基本问题之一一一原 子核中强相互作用的性质提供新的重要认识.

近些年轻核中的团簇结构又重新引起了人们的 极大兴趣. 一方面, 因为阈值附近的这些团簇结构对 于核天体物理中元素丰度及核合成具有决定性影响, 比如具有 $\alpha$ 团簇结构的 ${ }^{12} \mathrm{C}$ 第二个 $0^{+}$态 (Hoyle态)的存 在对恒星中的三 $\alpha$ 过程及 ${ }^{12} \mathrm{C} 、{ }^{16} \mathrm{O}$ 的丰度具有决定性 影响 ${ }^{[10]}$; 另一方面, 因为轻核是目前第一性原理计 算方法的主要研究对象, 比如格林函数蒙特卡洛方

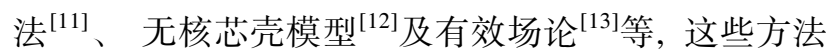
利用现实的核-核相互作用试图重现轻核中的团簇性 质, 理解Hoyle态的性质是目前这些理论所面临的首 要挑战, 对这个问题的深人理解需要更加细致地研 究Hoyle态的激发及衰变. 轻核中的团簇是个覆盖很 宽的领域, 且最近进展非常迅速 ${ }^{[10,14]}$, 本文主要集中 介绍最近 ${ }^{12} \mathrm{C}$ 和 ${ }^{16} \mathrm{O}$ 团簇研究的重要理论和实验进展.

\section{$1 \quad{ }^{12}$ C Hoyle态及其重要意义}

利用氦燃烧来解释 ${ }^{12} \mathrm{C}$ 和 ${ }^{16} \mathrm{O}$ 自然丰度问题时, Hoyle ${ }^{[15]}$ 年预言在7.68 MeV附近应存在 ${ }^{12} \mathrm{C}$ 的激发态, 随后的实验测量证实了在 $7.65 \mathrm{MeV}$ 存在一个 $0^{+}$激发 态 ${ }^{[16]}$, 这个态被称作Hoyle态. 由于Hoyle的存在, ${ }^{8} \mathrm{Be}$ 俘获 $\alpha$ 的概率增大了 7,8 个量级, 因此Hoyle态是宇宙
中碳合成及碳基生命存在的基础. Hoyle态的存在和 解释可以看作是人择宇宙学原理的一个典型例子, 虽然Hoyle做出这个预言时并没有依赖人择原理，而 是为了理解 ${ }^{12} \mathrm{C}$ 和 ${ }^{16} \mathrm{O}$ 的自然丰度. $7.65 \mathrm{MeV}$ 的 Hoyle 态位于 ${ }^{12} \mathrm{C}$ 的 $3 \alpha$ 衰变阈值 (7.367) 附近, $\alpha$ 团簇模

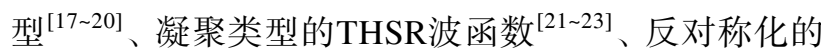

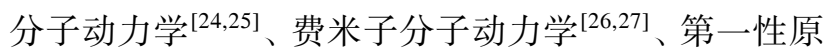
理计算 ${ }^{[13,28]}$ 及动力学对称方法 ${ }^{[29]}$ 等不同理论对Hoyle 态的研究都表明其具有发育良好的 $\alpha$ 团簇结构, 这和 恒星中 ${ }^{12} \mathrm{C}$ 主要通过 $3 \alpha$ 过程合成的假设是符合的, 其 中 $\alpha-\alpha$ 之间的关联起着重要作用. 虽然不同的理论模 型都认为Hoyle态比基态的尺寸大得多, 但是目前对 Hoyle态团簇性质的认识还有比较大的争议, 比如 $\alpha$ 团簇在其中是以玻色-爱因斯坦凝聚的形式存在，还 是 $\alpha$ 团簇气体态或是特定几何构型的 $\alpha$ 团簇结构. 所 以Hoyle态的存在不仅具有重要的现实意义, 对其合 理的描述也成为核天体物理, 核结构及核力研究的重 要试金石, 因此成为最近核中团簇研究的热点问题.

\section{$2 \alpha$ 团簇态理论描述}

\section{$2.1 \alpha$ 团簇模型}

$\alpha$ 团簇模型预先假设 $\alpha$ 共轭核是由 $\alpha$ 团簇构成, 每个 $\alpha$ 团簇的波函数可以表示为 


$$
\phi_{i}(\vec{r})=\sqrt{\frac{1}{b^{3} \pi^{3 / 2}}} \exp \left[\frac{-\left(\vec{r}-\vec{R}_{i}\right)^{2}}{2 b^{2}}\right],
$$

系统的波函数由每个 $\alpha$ 粒子的波函数反对成化 后得到

$$
\Phi_{\alpha}\left(\vec{R}_{1}, \vec{R}_{2}, \cdots, \vec{R}_{N}\right)=K \mathcal{A} \prod_{i=1}^{N} \phi_{i}\left(\vec{R}_{i}\right),
$$

其中 $\mathcal{A}$ 为反对成算符, $K$ 为归一化因子. 如果 $\alpha$ 团簇 离得比较近, 反对称化导致 $\alpha$ 被破坏, 当 $\alpha$ 团簇离得 比较远时， $\alpha$ 团簇的波色属性得以体现. $\alpha$ 团簇的最优 排列对应系统的最小能量, 通过改变 $\alpha$ 的位置及大小 得到. $\alpha$ 团簇模型曾预言 ${ }^{12} \mathrm{C}$ 基态为由 $\alpha$ 构成的正三角 形结构, Hoyle态是 $3 \alpha$ 构成的链状结构.

\subsection{THSR波函数}

非弹性电子散射测量表明 ${ }^{[30 \sim 32]}$ Hoyle态的半径比 基态大 $1.35 \sim 1.60$ 倍, 因此体积比基态大 $2.5 \sim 4$ 倍. 在 $\alpha$ 衰变阈值附近, 核的半径比基态大很多, 此时核中的 $\alpha$ 团簇具有玻色子的性质, 反对称化可能起得作用比 较小, 类比于原子系统, Hoyle态可能和 $\alpha$ 粒子的玻色爱因斯坦凝聚态有关. 因此Tohsaki, Horiuchi, Schuck 和Röpke采用Bloch-Brink类型的波函数来描述 ${ }^{12} \mathrm{C}$ 等 核中可能的团簇结构, 这种波函数被称作THSR波函 数, THSR波函数具有凝聚类型的特征, 系统总的波 函数由每个 $\alpha$ 团簇波函数反对称化后得到

$$
\begin{aligned}
\left\langle\vec{r}_{1}, \vec{r}_{2}, \cdots, \vec{r}_{N} \mid \Phi_{n \alpha}\right\rangle & \\
= & \mathcal{A}\left[\phi_{\alpha}\left(\vec{r}_{1}, \vec{r}_{2}, \vec{r}_{3}, \vec{r}_{4}\right) \phi_{\alpha}\left(\vec{r}_{5}, \vec{r}_{6}, \vec{r}_{7}, \vec{r}_{8}\right) \cdots\right. \\
& \left.\phi_{\alpha}\left(\vec{r}_{N-3}, \vec{r}_{N-2}, \vec{r}_{N-1}, \vec{r}_{N}\right)\right] .
\end{aligned}
$$

每个 $\alpha$ 的波函数分为质心部分和 $\alpha$ 内部两部分,

$$
\phi_{\alpha}\left(\vec{r}_{1}, \vec{r}_{2}, \vec{r}_{3}, \vec{r}_{4}\right)=\exp \left[-\frac{2\left(X_{i}-X_{G}\right)^{2}}{B^{2}}\right] \phi\left(\vec{r}_{1}-\vec{r}_{2}, \vec{r}_{1}-\vec{r}_{3}, \cdots\right),
$$

其中 $X_{i}$ 是 4 个核子的质心, $X_{G}$ 是所有核子的质心, $\phi$ 是 高斯型的波包,

$$
\phi\left(\vec{r}_{1}-\vec{r}_{2}, \vec{r}_{1}-\vec{r}_{3}, \cdots\right) \propto \exp \left[-\frac{1}{8 b^{2}} \sum_{k<l}\left(\vec{r}_{k}-\vec{r}_{l}\right)^{2}\right] .
$$

相比 $\alpha$ 团簇模型，除了表征自由 $\alpha$ 粒子的大小参 数外, 多了一个参数 $B$ 来描述多个 $\alpha$ 团簇的分布宽度, 在 $B>>b$ 时, 系统相当于自由 $\alpha$ 气体, 当 $B$ 和 $b$ 可比拟 时, 相当于壳模型的波函数. 此方法目前最大的成功 在于, 在没有任何自由归一化的因子下, 能非常好地 重现了利用非弹性电子散射把 ${ }^{12} \mathrm{C}$ 从基态散射到 Hoyle态的电荷形状因子的实验数据. 对实验数据的
成功描述暗示Hoyle态具有相当大的空间扩展以及具 有发育良好的 $\alpha$ 团簇自由度.

THSR 得到的Hoyle态波函数和 3 个 $\alpha$ 都处在 $S$ 态

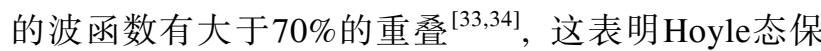
持了 $\alpha$ 粒子的渐近性质, 此时 Hoyle态很大程度上具 有玻色-爱因斯坦 (BEC) 凝聚波函数的性质. 类比于 ${ }^{12} \mathrm{C}$ Hoyle态，THSR波函数预言 ${ }^{16} \mathrm{O}$ 的 $0_{6}^{+}$态也具有类 似Hoyle态的凝聚性质, 实验上观测到的 $15.1 \mathrm{MeV}$ 的 态可能和THSR的预言相符合，同时四体正交条件模 型也预言 $0_{6}^{+}$态具有 $\alpha$ 凝聚态的性质 ${ }^{[35]}$. 相反对 ${ }^{12} \mathrm{C}$ 基 态的描述, $S, D, G$ 不同分波具有差不多相同的成 分 ${ }^{[33,34]}$, 表明在基态中泡利不相容原理破坏了 $\alpha$ 粒 子. 包含 $2 \alpha$ 关联的THSR计算表明基态中的 $\alpha-\alpha$ 关联 比Hoyle态中的 $\alpha-\alpha$ 关联要强 ${ }^{[36]}$.

通过改进的角动量投影的 THSR波函数 ${ }^{[37]}$ 对 ${ }^{20} \mathrm{Ne}$ inversion-doublet带的研究表明 $K^{\pi}=0^{-}$和 $K^{\pi}=0^{+}$的带具 有非定域团簇的特性 ${ }^{[38]}$, 团簇是在整个核的体积内 运动而非定域的. THSR波函数揭示 ${ }^{12} \mathrm{C}$ 和 ${ }^{16} \mathrm{O}$ 一维 $\alpha$ 链 状态具有 $\alpha$ 凝聚的性质 ${ }^{[39]}$, 但由于 Pauli不相容原理 引起的排斥导致密度等高图显示出定域的 $\alpha$ 团簇特 征. 因此也通常把 THSR波函数称作 BEC类型的波 函数.

\section{3 分子动力学方法}

反对称分子动力学(AMD)由传统分子动力学发 展而来, 由于采用Slater行列式来描述系统的波函数, 表示为

$$
\phi=\frac{1}{\sqrt{A !}} \mathcal{A}\left\{\varphi_{1}, \varphi_{2}, \cdots, \varphi_{A}\right\},
$$

因此系统精确地符合Pauli不相容原理并能合理地描 述核子的费米子属性. $\varphi_{i}$ 为高斯波包并考虑核子的自 旋和同位旋量子数，系统哈密顿量由有效核子-核子 相互作用得到，演化方程由变分原理得到．和团簇模 型相比，AMD模型基于微观核子自由度，不预先假 定团簇自由度的存在, AMD波函数的灵活性使其 能够同时对壳结构和团簇结构给出合理的描述, 壳或团簇自由度的出现依赖于细致的核子-核子相互 作用.

如图2所示, AMD模型不仅能够相当好地重现 ${ }^{12} \mathrm{C}$ Hoyle态的能量, 而且能够重现國值下的类壳结 构 $2^{+}$态的能量, 而团簇模型比如共振群方法 ${ }^{[40]}$ 和生 成坐标方法 ${ }^{[41 ~ 43]}$ 虽然能够描述阈值以上团簇自由度 


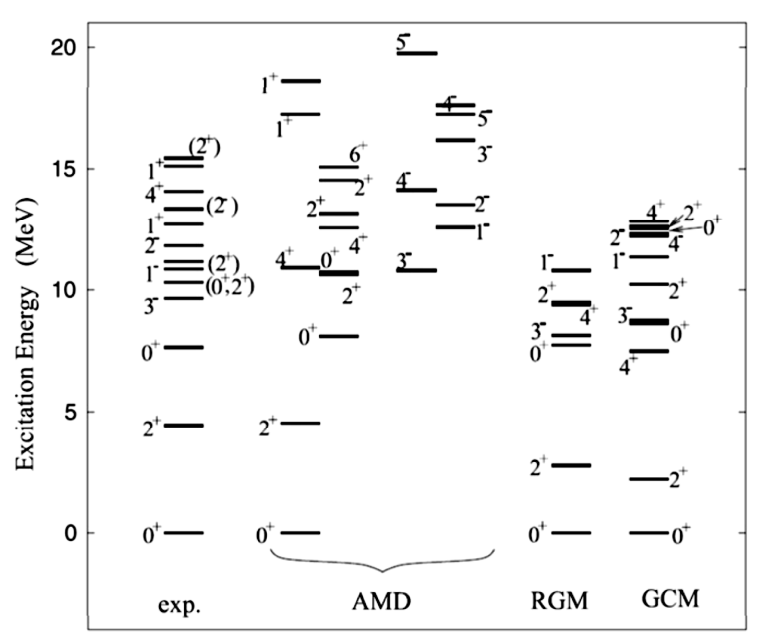

图 2 反对称化分子动力学 ${ }^{[25]}$ 、共振群方法 ${ }^{[40]}$ 和生成坐标方法 ${ }^{[41 ~ 43]}$ 对 ${ }^{12} \mathrm{C}$ 激发谱的描述以及和实验数据的对比 ${ }^{[25]}$

Figure 2 The comparison of excitation spectrum calculated by antisymmetrised molecular dynamics(AMD) approach $^{[25]}$, resonating group method $(\mathrm{RGM})^{[40]}$ and generator coordinate method $(\mathrm{GCM})^{[41-43]}$ against experimental data ${ }^{[25]}$

比较强的态, 但并不能描述 $2^{+}$的能量. AMD模型对 ${ }^{12} \mathrm{C}$ 计算表明基态是个紧致的结构, Hoyle态是个相当 扩展的 $\alpha$ 团簇结构.

费米子分子动力学 (FMD) 模型 ${ }^{[26,27]}$ 除了具有 $\mathrm{AMD}$ 模型的特征外, 还引人了可变的波包宽度作为 另外的自由度, 和 AMD模型一样, FMD模型同样能 够很好地重现 ${ }^{12} \mathrm{C} 0_{1}^{+} \rightarrow 0_{2}^{+}$散射的电荷形状因子, 但 是Hoyle态并不是由单一的结构组成, 可以解释为不 同构型的叠加, 相反, 基态具有单一的成分. 但是 FMD预言的Hoyle态比实验值高了 $2 \mathrm{MeV}$.

最近, 我们利用扩展的量子分子动力学方法 $(\mathrm{EQMD}){ }^{[44 \sim 46]}$ 能很好地构建 ${ }^{8} \mathrm{Be},{ }^{12} \mathrm{C}$ 及 ${ }^{16} \mathrm{O}$ 等不同 $\alpha$ 团 簇构型, 发现它们对应的巨偶极共振谱 $(G D R)^{[47 \sim 49]}$ 与 $\alpha$ 团簇的几何构型存在着很强的对应. 从与实验数 据的定量比较来看, 利用 ${ }^{16} \mathrm{O}$ 四面体的 $4 \alpha$ 构型能很好 地拟合 ${ }^{16} \mathrm{O}$ 的GDR谱. 图3给出了利用EQMD模型得 到的 ${ }^{8} \mathrm{Be},{ }^{12} \mathrm{C}$ 和 ${ }^{16} \mathrm{O}$ 不同 $\alpha$ 团簇几何构型的中子质心、质 子质心沿着长轴与短轴振荡的GDR谱. $30 \mathrm{MeV}$ 附近 红色的谱说明了不管 $\alpha$ 团簇的几何构型多么不同, 沿 着短轴方向它们有着共同的振荡谱, 因此它是判断 轻核是否有 $\alpha$ 团簇结构的探针; 而沿着长轴方向, 不 同的几何构型显示了丰富的GDR结构, 这与动力学 对称性和几何构型紧密相关. 因此巨偶极共振谱可 以作为团簇构型的强有力探针 ${ }^{[50,51]}$. 当然, 要测量建

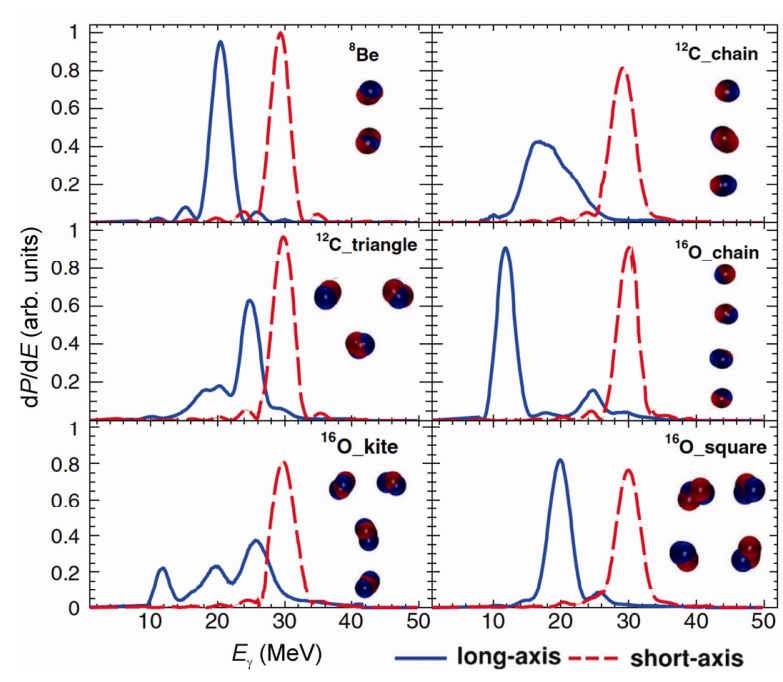

图 3 (网络版彩色)利用 $\mathrm{EQMD}$ 模型计算得到的 ${ }^{8} \mathrm{Be},{ }^{12} \mathrm{C}$ 和 ${ }^{16} \mathrm{O}$ 不同 $\alpha$ 团簇的几何构型对应的沿着短轴(红色虚线)与长轴(蓝色实线)方向振 荡的巨偶极共振谱, 其中, 短轴指垂直于团簇平面或团簇链的方向, 长轴指在团簇平面内或与团簇链平行的最长构型的方向, 图中的团簇 构型由 EQMD 初始化得到, 蓝色球和红色球分别代表质子和中子 ${ }^{[50]}$

Figure 3 (Color online) The giant resonant dipole (GDR) spectrum corresponding to different cluster configurations of ${ }^{8} \mathrm{Be},{ }^{12} \mathrm{C}$ and ${ }^{16} \mathrm{O}$ along short axis (red dashed line) and long axis (blue solid line) calculated by an extended quantum molecular dynamics (EQMD) model, where short axis is perpendicular to the plane or the chain of the $\alpha$ configurations and long axis is the longest axis of the configuration in the $\alpha$ cluster plane or along the $\alpha$ chain. The $\alpha$ cluster configuration is obtained by the initialization of EQMD model, where the blue and red balls indicate protons and neutrons, respectively ${ }^{[50]}$

立在 $\alpha$ 团簇激发态构型上的巨偶极共振需要高精度 的带电粒子、中子及 $\gamma$ 等的多举符合测量, 以鉴别高 激发的 $\alpha$ 团簇的不同衰变道及电磁跃迁.

\section{4 从头算起方法}

和AMD及FMD采用有效核子-核子相互作用不 同，从头计算方法基于第一性原理，采用裸核子-核 子相互作用, 从而试图重现有限核性质, 尤其是轻 核. 怎么把从 $n-p$ 两核子系统和核子-核子散射中得到 核子-核子相互作用应用到有限核中是目前遇到的很 大挑战. 单介子交换或许并不能解释核子-核子相互 作用的复杂性, 三体及高阶项将会起很大作用.

Wiringa等人 ${ }^{[52]}$ 利用格林函数蒙特卡洛方法计算了 为什么 $A=5$ 和 $A=8$ 的核不存在, 发现其严重依赖于核力 的自旋、同位旋及张量成分, 但这种方法在三体相互作 用的处理上还具有较大的不确定性. 手征有效场论能 够自洽地处理两体及多体相互作用，三体、四体及更高 阶相互作用通过NNLO及NNNLO等项自然出现 ${ }^{[53]}$. 
Epelbaum等人 ${ }^{[13,28,54]}$ 利用格点手征有效场论计 算了 $A=3,4$ 和 6 的核及 ${ }^{12} \mathrm{C}$ 的基态、Hoyle态, 虽然格点 取得相当粗糙, 但能够相当好地重现这些态的结合 能. Epelbaum等人 ${ }^{[55]}$ 同样计算了夸克质量对 $\alpha$ 结合能 及恒星中 $3 \alpha$ 过程的影响. 最近Epelbaum等人 ${ }^{[56]}$ 把手 征有效场论计算扩展到了更重的 ${ }^{16} \mathrm{O}$, 很好地重现了 ${ }^{16} \mathrm{O}$ 的能谱及电磁性质, 同时发现 ${ }^{16} \mathrm{O}$ 基态是一个正四 面体的 $\alpha$ 结构, 第一激发态是正方形的 $\alpha$ 结构.

基于 $\mathrm{QCD}$ 得到的相互作用被应用到无核壳模型 中 $^{[57]}$, 无核壳模型能对一系列轻核给出相当满意的描 述, 且能够相当好地描述 ${ }^{8} \mathrm{Be}$ 的 $\alpha$ 团簇结构 ${ }^{[58]}$, 但并不 能给出正确的Hoyle态能量 ${ }^{[59]}$, 这说明无核壳模型采 用的谐振子基不太适合用来描述弱束缚系统的行为.

\section{5 动力学对称模型}

早在1938年Hafstad和Teller就提出 ${ }^{2} \mathrm{C}$ 中可能存在 和 $3 \alpha$ 相联系的动力学对称性. ${ }^{12} \mathrm{C}$ 基态具有负的四极 形变, $\alpha$ 团簇的成分较小, $\alpha$ 团簇的谱因子和壳模型的 计算相符. 虽然 ${ }^{12} \mathrm{C}$ 基态没有明显的 $\alpha$ 团簇结构, 但依 然具有 $3 \alpha$ 结构的对称性. 绕着 ${ }^{12} \mathrm{C}$ 基态在平面内的旋 转激发可以布局到 $0^{+}, 2^{+}, 4^{+}$等态, 绕着垂直于在平面 轴的集体激发可以布局到 $3^{-}, 4^{-}, 5^{-}$等态, 对应 $K=0$, $K=3$ 的带. 旋转谱和 $D_{3 \mathrm{~h}}$ 点群对称紧密联系, 如果加 上振动谱, 可表示为 ${ }^{[60,61]}$

$$
E=E_{0}+A v_{1}+B v_{2}+C L(L+1)+D(K \pm 2 l)^{2},
$$

其中 $v_{1}$ 和 $v_{2}$ 是振动量子数, $L$ 为角动量, $K$ 为角动量投 影, $A, B, C, D$ 为拟合参数. 通过(7)式中不同的旋转谱 可以得出有关团簇几何分布的信息.

\section{$3 \alpha$ 团簇态实验测量}

测量 $\alpha$ 衰变阈值之上的激发是辨别各种团簇构 型的重要方法. ${ }^{12} \mathrm{C}$ 的各种激发谱如图4所示 ${ }^{[62]}$, 其中 ${ }^{12} \mathrm{C}$ 的基态带对应 $K=0$, 其中 $0^{+}$(基态), $2^{+}(4.4 \mathrm{MeV}$ )和 $4^{+}(14.1 \mathrm{MeV})$ 已经认识的比较清楚了. 最近, MarínLámbarri等人 ${ }^{[62]}$ 测量到了位于 22.4(2) $\mathrm{MeV}$ 的 $J^{\pi}=5^{-}$态, $5^{-}$态的测量非常明确证实了 ${ }^{12} \mathrm{C}$ 基态的 $\mathrm{D}_{3}$ 对称性. Yang等人 ${ }^{[63]}$ 通过测量 ${ }^{12} \mathrm{Be}$ 衰变到 ${ }^{4} \mathrm{He}+{ }^{8} \mathrm{He}$ 的道确认 $3^{12} \mathrm{Be} 10.3 \mathrm{MeV}$ 激发能附近的 $0^{+}$态, 此共振态具有 大的单极跃迁强度和团簇衰变分支比, 这些证据支 持 ${ }^{12} \mathrm{Be}$ 具有强的团簇结构.

类似Hoyle态中 $\alpha$ 团簇的分布也可以从建立在 Hoyle态上的旋转带导出, 经过众多实验的努力, 最

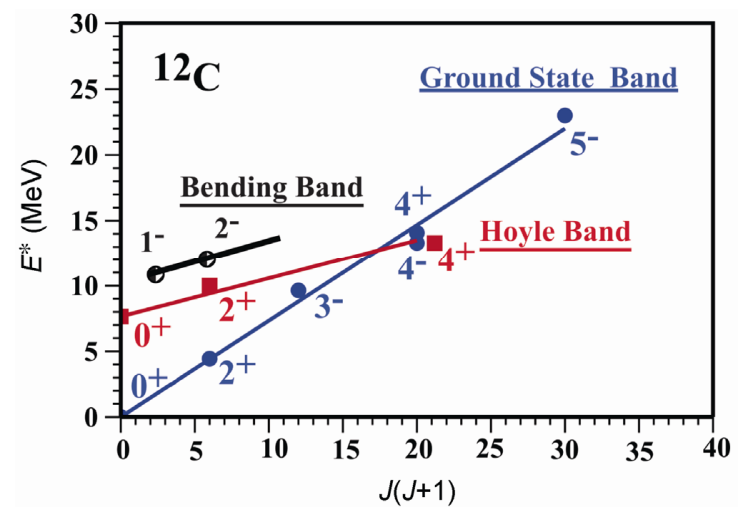

图 4 (网络版彩色) $)^{12} \mathrm{C}$ 的基态带, Hoyle 带及弯曲振动带 ${ }^{[62]}$

Figure 4 (Color online) Rotational band structure of the ground band, the Hoyle band and the bending vibration band of ${ }^{12} \mathrm{C}^{[62]}$

近 $\mathrm{HI} \gamma \mathrm{S}$ 通过 ${ }^{12} \mathrm{C}(\gamma, 3 \alpha)$ 反应的实验清楚地认定了 ${ }^{12} \mathrm{C}$ 的 $J^{\pi}=2^{+}$的能量为 $10.03(11) \mathrm{MeV}^{[64]}$, 作为 Hoyle态的激 发, 此数据很大程度上排除了 Hoyle态为链状态的可 能性 ${ }^{[64 ~ 66]}$, 各种三角形分布的 $\alpha$ 构型存在较大的可能 性. 如果按照 $J(J+1)$ 的标度, Hoyle态的下一个激发态 应该是处在 $14 \mathrm{MeV}$ 的 $4^{+}$态, 但目前需要更多实验来 证实 $14 \mathrm{MeV}$ 的 $4^{+}$态 ${ }^{[67]}$. 但手征有效场论得到的 ${ }^{12} \mathrm{C}$ Hoyle态是近似链状的 ${ }^{[13]}$, 要得到一致的结论还需要 不同的方法.

Bijker和Iachello ${ }^{[68]}$ 最近通过代数模型对 ${ }^{16} \mathrm{O}$ 激发 谱的分析得出 ${ }^{16} \mathrm{O}$ 是由 $\alpha$ 组成的正四面体结构, 这个 结论和手征有效场论对 ${ }^{16} \mathrm{O}$ 基态的计算是相符的 ${ }^{[56]}$.

另一方面, 原则上Hoyle态的 $\alpha$ 衰变应该能够提 供 $\alpha$ 自由度及其存在状态的最直接的信息, 一些观点 认为衰变末态 $\alpha$ 分布和位于库伦位垒之内的 $\alpha$ 构型存 在一一对应关系。早在 20 世纪 50 年代 Cook等人就发 现通过 ${ }^{8} \mathrm{Be}$ 的相继衰变 ${ }^{12} \mathrm{C} \rightarrow \alpha+{ }^{8} \mathrm{Be}$ 是 Hoyle态的主 要衰变模式 ${ }^{[16]}$, 但直到 90 年代才得到Hoyle态直接衰

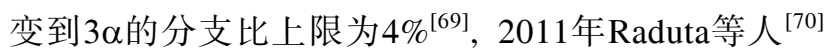
的测量给出了 $17 \%$ 的 $3 \alpha$ 同时衰变的分支比, 认为得 到的能量均分的 $3 \alpha$ 直接衰变可以作为Hoyle态存在 $\alpha$ 凝聚的直接证据. 如果Hoyle态为链状构型, 按照动 量守恒, 在 ${ }^{12} \mathrm{C}$ 质心系下链两端的两个 $\alpha$ 会带走全部 的衰变动能, 中间的 $\alpha$ 处于静止状态. Raduta等人的 测量结果引发了众多测量, 之后的测量否定了这一 结果, 得到的 $3 \alpha$ 同时衰变的分支比的上限为 $5 \%$ o $(95 \%$ 置信度 $)^{[71]}, 4.5 \% 0(99.75 \% \text { 置信度 })^{[72]}, 9 \% 0^{[73]}$, 最新的 测量给出 $2 \%$ o( $\left(95 \%\right.$ 置信度)的上限 ${ }^{[74]}$.

$\mathrm{Pei}$ 等人 ${ }^{[75]}$ 利用 $\mathrm{WKB}$ 方法研究了 $\mathrm{Be}$ 和 $\mathrm{C}$ 等同位素 
从激发态衰变到基态的衰变宽度，与实验符合得很 好, 但目前研究高激发 $3 \alpha$ 态同时衰变动力学的工作 还相对较少, 怎么解释如此小的 $3 \alpha$ 衰变分支比在理 论上是一个很大的挑战, 比如衰变时的位垒穿透可 能会影响 $3 \alpha$ 的最初构型, 这需要不同的理论模型相 互验证 ${ }^{[76,77]}$.

\section{4 结语}

对 ${ }^{12} \mathrm{C}$ 的团簇结构虽然已经积累了不同类型的实 验数据并发展了各种理论模型, 各种理论都预言 Hoyle态是一个比基态尺寸大很多的 $\alpha$ 主导的团簇态, 这和对 ${ }^{12} \mathrm{C}$ 的非弹散射和从 $\alpha$ 衰变率中导出的 Hoyle态 大小是相符的. 但不同理论方法对 $\alpha$ 团簇在 Hoyle态 中的存在状态给出不同的描述, 比如 $\alpha$ 凝聚, $\alpha$ 气体或 是具有特定几何构型的 $\alpha$ 结构. 虽然比Hoyle态高 2 $\mathrm{MeV}$ 多的 $2^{+}$态已经被确认为具有较好的团簇结构, 但 $2^{+}$态的特征及它和Hoyle态的关系还需要仔细研究,
另外Hoyle的另外一个激发态一位于 $14 \mathrm{MeV}$ 附近的 $4^{+}$态也需要更多的实验确认.

测量 $\alpha$ 衰变阈之上的团簇态的偶极跃迁能够给 出这些团簇态结构的重要信息 ${ }^{[78]}$, 但这些测量目前 还非常困难. 在现有技术条件下，利用质子或 $\alpha$ 的非 弹性散射来布居团簇态, 通过其衰变 $\alpha$ 的关联测量能 够给出这些团簇结构及核天体物理一些重要启示.

最新理论方法对 ${ }^{16} \mathrm{O}$ 的计算认为其基态可由 $\alpha$ 才 簇构成的正四面体结构描述，同时理论也预言 ${ }^{16} \mathrm{O}$ 中 存在类似的Hoyle态, 显然对 ${ }^{16} \mathrm{O} \alpha$ 衰变阈值之上团簇 结构的认识更加困难.

综上所述, 即便对最简单的 ${ }^{12} \mathrm{C}$, 我们对其团簇 结构的理解还有很长的路要走, 任何实质性的进展 都需要精确的实验测量和各种最新的、可靠的理论高 度结合. ${ }^{12} \mathrm{C}$ 团簇结构丰富且精确的数据将成为核力 及最新核多体理论的试金石, 也为核天体物理研究 提供重要输人.

\section{参考文献}

1 Hafstad L R, Teller E. The alpha-particle model of the nucleus. Phys Rev, 1938, 54: 681-692

2 Ikeda K, Takigawa N, Horiuchi H. The systematic structure-change into the molecule-like structures in the self-conjugate $4 \mathrm{n}$ nuclei. Prog Theor Phys (Supplement), 1968, E68: 464-475

3 Girod M, Schuck P. $\alpha$-particle clustering from expanding self-conjugate nuclei within the Hartree-Fock-Bogoliubov approach. Phys Rev Lett, 2013, 111: 132503

4 Tseng C Y, Cheng T S, Yang F C. Further study on nuclear mass formulas. Nucl Phys A, 1980, 334: 470-476

5 Ren Z Z, Xu G O. Evidence of a correlation from binding energies in medium and heavy nuclei. Phys Rev C, 1988, $38: 1078$

6 Ren Z Z, Xu G O. Short-range correlations in light nuclei near the neutron drip line. Phys Lett B, 1990, 237: 1-2

7 Ebran J P, Khan E, Niksic T, et al. How atomic nuclei cluster. Nature, 2012, 487: 341-344

8 Freer M. Nucleons come together. Nature, 2012, 487: 309-310

9 von Oertzen W, Freer M, Kanada-En'yo Y. Nuclear clusters and nuclear molecules. Phys Rep, 2006, 432: 43-113

10 Freer M, Fynbo H O U. The Hoyle state in ${ }^{12}$ C. Prog Part Nucl Phys, 2014, 78: 1-23

11 Wiringa R B, Pieper S C, Pandharipande V R. Quantum Monte Carlo calculations of A=8 nuclei. Phys Rev C, 2000, 62: 014001

12 Navrátil P, Vary J P, Barrett B R. Properties of ${ }^{12} \mathrm{C}$ in the Ab Initio nuclear shell model. Phys Rev Lett, 2000, 84: 5728

13 Epelbaum E, Krebs H, Lee D, et al. Ab Initio calculation of the Hoyle state. Phys Rev Lett, 2011, 106: 192501

14 Freer M. Challenges to the field of nuclear clustering. J Phys Conf Ser, 2013, 436: 012002

15 Hoyle F. On nuclear Reactions occuring in very hot STARS.I. the synthesis of elements from carbon to nickel. Astron Astrophys Sup, 1954, 1: 121-146

16 Cook C W, Fowler W A, Lauritsen C C, et al. ${ }^{12} \mathrm{~B},{ }^{12} \mathrm{C}$, and the red giants. Phys Rev, 1957, 107: 508

17 Marenau H. Interaction of Alpha-particles. Phys Rev, 1941, 59: 37

18 Brink D. Many-body description of nuclear structure and reactions. In: Bloch C, ed. Proceedings of the International School of Physics "Enrico Fermi", Course 36. London: Academic Press, 1966. 247-277

19 Brink D M. Boeker E. Effective interactions for Hartree-Fock calculations. Nucl Phys A, 1967, 91: 1-26

20 Brink D M. History of cluster structure in nuclei. J Phys Conf Ser, 2008, 111: 012001

21 Tohsaki A, Horiuchi H, Schuck P, et al. Alpha cluster condensation in ${ }^{12} \mathrm{C}$ and ${ }^{16} \mathrm{O}$. Phys Rev Lett, 2001, 87: 192501

22 Funaki Y, Tohsaki A, Horiuchi $\mathrm{H}$, et al. Inelastic form factors to alpha-particle condensate states in ${ }^{12} \mathrm{C}$ and ${ }^{16} \mathrm{O}$ : What can we learn? Eur Phys J A, 2006, 28: 259-263 
23 Funaki Y, Tohsaki A, Horiuchi H, et al. Analysis of previous microscopic calculations for the second $0^{+}$state in ${ }^{12} \mathrm{C}$ in terms of $3-\alpha$ particle Bose-condensed state. Phys Rev C, 2003, 67: 051306(R)

24 Kanada En'yo Y, Horriuchi H. Structure of Light unstable nuclei studied with antisymmetrized molecular dynamics. Prog Theor Phys Supp, 2001, 142: 205-263

25 Kanada En'yo Y. The structure of ground and excited states of ${ }^{12} \mathrm{C}$. Prog Theor Phys, 2007, 117: 655-680

26 Chernykh M, Feldmeier H, Neff T, et al. Structure of the Hoyle state in ${ }^{12}$ C. Phys Rev Lett, 2007, $98: 032501$

27 Roth R, Neff T, Feldmeier H. Nuclear structure in the framework of the unitary correlation operator method. Prog Part Nucl Phys, 2010, 65: 50-93

28 Epelbaum E, Krebs H, Lähde T A, et al. Structure and rotations of the Hoyle state. Phys Rev Lett, 2012, 109: 252501

29 Bijker R, Iachello F. Cluster states in nuclei as representations of a U(v+1) group. Phys Rev C, 2000, 61: 067305

30 Sick I, McCarthy J S. Elastic electron scattering from ${ }^{12} \mathrm{C}$ and ${ }^{16} \mathrm{O}$. Nucl Phys A, 1970, 150: 631-654

31 Nakada A, Torizuka Y, Horikawa Y. Determination of the Deformation in ${ }^{12} \mathrm{C}$ from Electron Scattering. Phys Rev Lett, 1971, 27: 745; Phys Rev Lett, 1971, 27: 1102, Erratum

32 Strehl P, Schucan Th H. Study of monopole transitions in ${ }^{12} \mathrm{C},{ }^{24} \mathrm{Mg},{ }^{28} \mathrm{Si},{ }^{32} \mathrm{~S}$ and ${ }^{40} \mathrm{Ca}$ by inelastic electron scattering. Phys Lett B, 1968 , 27: $641-643$

33 Funaki Y, Horiuchi H, von Oertzen W, et al. Concepts of nuclear $\alpha$-particle condensation. Phys Rev C, 2009,80 : 064326

34 Yamada T, Schuck P. Single alpha-particle orbits and Bose-Einstein condensation in ${ }^{12}$ C. Eur Phys J A, 2005, 26: 185-199

35 Funaki Y, Yamada T, Horiuchi H, et al. $\alpha$-Particle condensation in ${ }^{16} \mathrm{O}$ studied with a full four-body orthogonality condition model calculation. Phys Rev Lett, 2008, 101: 082502

36 Zhou B, Funaki Y, Tohsaki A, et al. The container picture with two-alpha correlation for the ground state of ${ }^{12} \mathrm{C}$. Prog Theor Exp Phys, 2014: 101D01

37 Zhou B, Ren Z Z, Xu C, et al. New concept for the ground-state band in ${ }^{20}$ Ne within a microscopic cluster model. Phys Rev C, 2012, 86: 014301

38 Zhou B, Funaki Y, Horiuchi H, et al. Nonlocalized clustering: A new concept in nuclear cluster structure physics. Phys Rev Lett, 2013, 110: 262501

39 Suhara T, Funaki Y, Zhou B, et al. One-dimensional $\alpha$ condensation of $\alpha$-linear-chain states in ${ }^{12} \mathrm{C}$ and ${ }^{16} \mathrm{O}$. Phys Rev Lett, 2014 , 112 : 062501

40 Kamimura M. Transition densities between the $0_{1}^{+}, 2_{1}^{+}, 4_{1}^{+}, 0_{2}^{+}, 2_{2}^{+}, 1_{1}^{-}$and $3_{1}^{-}$states in ${ }^{12} \mathrm{C}$ derived from the three-alpha resonating-group wave functions. Nucl Phys A, 1981, 351: 456-480

41 Uegaki E, Okabe S, Abe Y, et al. Structure of the excited states in ${ }^{12}$ C. I. Prog Theor Phys, 1977, 57: 1262-1276

42 Uegaki E, Abe Y, Okabe S, et al. On the positive-parity states with anomalous $\alpha$-decay properties in ${ }^{12}$ C. Prog Theor Phys, 1978 , 59: $1031-1033$

43 Uegaki E, Abe Y, Okabe S, et al. Structure of the excited states in ${ }^{12}$ C. II. Prog Theor Phys, 1979, 62: 1621-1640

44 Maruyama T, Niita K, Iwamoto A. Extension of quantum molecular dynamics and its application to heavy-ion collisions. Phys Rev C, 1996, 53: 297-304

45 Wada R, Hagel K, Cibor J, et al. Entrance channel dynamics in ${ }^{40} \mathrm{Ca}+{ }^{40} \mathrm{Ca}$ at $35 \mathrm{~A} \mathrm{MeV}$. Phys Lett B, 1998, 422: 6-12

46 Cao X G, Ma Y G, Zhang G Q, et al. Role of wave packet width in quantum molecular dynamics in fusion reactions near barrier. J Phys Conf Ser, 2014, 515: 012023

47 Wu H L, Tian W D, Ma Y G, et al. Dynamical dipole $\gamma$ radiation in heavy-ion collisions on the basis of the quantum molecular dynamics model. Phys Rev C, 2010, 81: 047602

48 Tao C, Ma Y G, Zhang G Q, et al. Symmetry energy dependence of the pygmy and giant dipole resonances in an isospin dependent quantum molecular dynamics model. Nucl Sci Tech, 2013, 24: 030502

49 Ye S Q, Cai X Z, Fang D Q, et al. Systematic study of dynamical dipole mode via the isospin-dependent Boltzmann-Uehling-Uhlenbeck model. Nucl Sci Tech, 2014, 25: 030501

50 He W B, Ma Y G, Cao X G, et al. Giant dipole resonance as a fingerprint of $\alpha$ clustering configurations in ${ }^{12} \mathrm{C}$ and ${ }^{16} \mathrm{O}$. Phys Rev Lett, 2014, 113: 032506

51 He W B, Cao X G, Ma Y G, et al. Application of EQMD model to researches of nuclear exotic structures. Nucl Tech, 2014, 37: 100511

52 Wiringa R B, Pieper Steven C. Evolution of nuclear spectra with nuclear forces. Phys Rev Lett, 2002, 89: 182501

53 Bernard V, Meißner Ulf-G. Chiral perturbation theory. Annu Rev Nucl Part Sci, 2007, 57: 33-60

54 Epelbaum E, Krebs H, Lee D, et al. Lattice effective field theory calculations for A=3, 4, 6, 12 nuclei. Phys Rev Lett, 2010, 104: 142501

55 Epelbaum E, Krebs H, Lähde Timo A, et al. Viability of carbon-based life as a function of the light quark mass. Phys Rev Lett, 2013, 110: 112502

56 Epelbaum E, Krebs H, Lähde Timo A, et al. Ab Initio calculation of the spectrum and structure of ${ }^{16}$ O. Phys Rev Lett, 2014 , $112: 102501$

57 Navrátil P, Quaglioni S, Stetcu I, et al. Recent developments in no-core shell-model calculations. J Phys G, 2009, 36: 083101 
58 Maris P, Vary J P, Navrátil P. Structure of A=7-8 nuclei with two- plus three-nucleon interactions from chiral effective field theory. Phys Rev C, 2013, 87: 014327

59 Navrátil P, Vary J P, Barrett B R. Properties of ${ }^{12} \mathrm{C}$ in the Ab Initio nuclear shell model. Phys Rev Lett, 2000, $84: 5728$

60 Bijker R, Iachello F, Leviatan A. Algebraic models of hadron structure. I. nonstrange baryons. Ann Phys, 1994, 236: 69-116

61 Bijker R, Dieperink A E L, Leviatan A. Spectrum-generating algebra for X 3 molecules. Phys Rev A, 1995, 52: 2786

62 Marín-Lámbarri D J, Bijker R, Freer R, et al. Evidence for triangular $\mathrm{D}_{3 \mathrm{~h}}$ symmetry in ${ }^{12} \mathrm{C}$. Phys Rev Lett, 2014, 113: 012502

63 Yang Z H, Ye Y L, Li Z H, et al. Observation of enhanced monopole strength and clustering in ${ }^{12}$ Be. Phys Rev Lett, 2014, $112: 162501$

64 Zimmerman W R, Ahmed M W, Bromberger, et al. Unambiguous identification of the second $2^{+}$state in ${ }^{12} \mathrm{C}$ and the structure of the hoyle state. Phys Rev Lett, 2013, 110: 152502

65 Itoh M, Akimune H, Fujiwara M, et al. Study of the cluster state at $E_{x}=10.3 \mathrm{MeV}$ in ${ }^{12} \mathrm{C}$. Nucl Phys A, 2004, 738: 268-272

66 Zimmerman W R, Destefano N E, Freer M, et al. Further evidence for the broad $2_{2}^{+}$state at $9.6 \mathrm{MeV}$ in ${ }^{12} \mathrm{C}$. Phys Rev C, 2011, 84: 027304

67 Freer M, Almaraz-Calderon S, Aprahamian A, et al. Evidence for a new ${ }^{12} \mathrm{C}$ state at $13.3 \mathrm{MeV}$. Phys Rev C, 2011, 83: 034314

68 Bijker R, Iachello F. Evidence for tetrahedral symmetry in ${ }^{16}$ O. Phys Rev Lett, 2014, 112: 152501

69 Freer M, Wuosmaa A H, Betts R R, et al. Limits for the $3 \alpha$ branching ratio of the decay of the $7.65 \mathrm{MeV}, \mathrm{O}_{2}{ }^{+}$state in ${ }^{12} \mathrm{C}$. Phys Rev C, 1994, 49: R1751(R)

70 Raduta Ad R, Borderie B, Geraci E, et al. Evidence for $\alpha$-particle condensation in nuclei from the Hoyle state deexcitation. Phys Lett B, 2011, 705: 65-70

71 Kirsebom O S, Alcorta M, Borgeet M J G, et al. Improved limit on direct $\alpha$ decay of the Hoyle state. Phys Rev Lett, 2012, 108: 202501

72 Manfredi J, Charity R J, Mercurio K, et al. $\alpha$ decay of the excited states in ${ }^{12} \mathrm{C}$ at 7.65 and $9.64 \mathrm{MeV}$. Phys Rev C, 2012, 85: 037603

73 Rana T K, Bhattacharya S, Bhattacharya C, et al. Estimation of direct components of the decay of the Hoyle state. Phys Rev C, 2013, 88: 021601(R)

74 Itoh M, Ando S, Aoki T, et al. Further improvement of the upper limit on the direct $3 \alpha$ decay from the Hoyle state in ${ }^{12} \mathrm{C}$. Phys Rev Lett, 2014, 113: 102501

75 Pei J C, Xu F R. Helium-cluster decay widths of molecular states in beryllium and carbon isotopes. Phys Lett B, 2007, 650: 224-228

76 Alvarez-Rodriguez R, Jensen A S, Fedorov D V, et al. Energy distributions from three-body decaying many-body resonances. Phys Rev Lett, 2007, 99: 072503

77 Fynbo H O U, Álvarez-Rodríguez R, Jensen A S, et al. Three-body decays and R-matrix analyses. Phys Rev C, 2009, 79: 054009

78 Datar V M, Chakrabarty D R, Suresh Kumar, et al. Electromagnetic transition from the $4^{+}$to $2^{+}$resonance in ${ }^{8}$ Be measured via the radiative capture in ${ }^{4} \mathrm{He}+{ }^{4} \mathrm{He}$. Phys Rev Lett, 2013, 111: 062502

\title{
Progress of theoretical and experimental studies on $\alpha$ cluster structures in light nuclei
}

\author{
CAO XiGuang $^{1} \&$ MA YuGang ${ }^{1,2}$ \\ ${ }^{1}$ Shanghai Institute of Applied Physics, Chinese Academy of Sciences, Shanghai 201800, China; \\ ${ }^{2}$ School of Physical Science and Technology, ShanghaiTech University, Shanghai 200031, China
}

The degree of freedom of $\alpha$ cluster exists widely in light $\alpha$ conjugate nuclei, which not only plays essential roles in the synthesis of elements and abandance of elements in nuclear astrophysics but also serves as the natural laboratory to test various new many-body theories and nuclear force due to the available high precision data. This article briefly summarizes the different theoretical approaches for alpha-cluster and compares the results of ${ }^{12} \mathrm{C}$ Hoyle state with these approaches. All the theories support the structure of Hoyle state with a more extended size compared with ground state. However, a consensus has not be reached about how $\alpha$ clusters stay inside Hoyle state, where several candidate states are possible, such as $\alpha$ condensate state, $\alpha$ gas or crystalline arrangements of the $\alpha$-particles. Further more, we also introduce the new experimental measurements of $\alpha$ decay and collective excitation of rotational band. In order to pin down the existence form of $\alpha$ in Hoyle state, a deeper knowledge of the collective excitation of cluster is needed. The essential progress can only be possible by high combination of new data with high precision and new many-body theory, where the roles of nucleon-nucleon correlation, cluster-cluster correlation and continuum are needed to be included reasonably.

\section{$\alpha$ cluster, $\alpha$ conjugate nucleus, Hoyle state, nuclear synthesis, Bose-Einstein condensation}

